\title{
Interações entre parasitoides e insetos endófagos em frutos de Stryphnodendron adstringens (Mart.) Coville (Fabaceae) no Cerrado Mineiro
}

Tiago Morales Silva ${ }^{1}$

Sônia Lúcia Modesto Zampieron²

\section{Resumo}

Os parasitoides são importantes controladores das populações de insetos fitófagos, responsáveis por diversos danos à planta, podendo vir a afetar sua sobrevivência e a dispersão de suas sementes. A biodiversidade dessa fauna parasitoide tem sido comprometida pelo avanço de atividades agropecuárias, principalmente no Cerrado. 0 objetivo deste estudo foi identificar a entomofauna parasítica e suas interações com insetos que se alimentam de frutos e sementes de S. adstringens, uma planta típica do Cerrado, conhecida como Barbatimão. Foram realizadas coletas mensais dos frutos durante o período de frutificação, de março a outubro de 2013, em uma área de Cerrado localizada no entorno do Parque Nacional Serra da Canastra (PNSC), no sudoeste mineiro. Representantes das ordens Coleoptera e Lepidoptera foram registrados como principais endófagos. Entre os parasitoides, destacou-se a família Braconidae, com representantes dos gêneros Psedophanerotoma, Chelonus, Hormius, Apanteles e Clinocentrus associados a lepidópteros e exemplares do gênero Allorhogas, cujo hábito é fitófago (endófago). O estudo mostrou, ainda, que algumas famílias de himenópteros parasitoides possuem ampla rede de relações ecológicas, tornando necessários estudos aprimorados.

Palavras-chave: Hymenoptera. Parasitoidismo. Relações tritóficas. Barbatimão. Cerrado.

\section{Introdução}

As plantas sofrem constantemente danos causados por insetos fitófagos. Tais insetos podem ser agrupados de acordo com a parte vegetal da qual se alimentam: raiz, caule, folha, flor, frutos ou sementes. Ao se alimentarem das estruturas reprodutivas das plantas, esses insetos comprometem a perpetuação dessa espécie, atuando como fator chave na mortalidade das sementes, podendo limitar a oferta delas ou mesmo impedir sua germinação; têm-se, portanto, consequências na trajetória, riqueza, diversidade e equitabilidade da distribuição das plantas (ZHANG et al., 1997).

Entre os insetos encontrados nos frutos, a entomofauna endofítica compreende os consumidores da polpa; polpa e sementes ou apenas das sementes (SARI; RIBEIRO-COSTA, 2011). As sementes representam um alimento potencial para animais herbívoros que buscam tecidos ricos em nutrientes (JANZEN, 1980), uma vez que contêm substâncias amiláceas, proteicas, oleaginosas ou celulósicas em suas estruturas internas (BARROSO et al., 1999).

\footnotetext{
1 Universidade Federal de Lavras, Programa de Pós-graduação em Entomologia. Lavras, MG, Brasil. tiagomorales.bio@hotmail.com, Caixa Postal 3037. Lavras, Minas Gerais, Brasil. Rua Júlio Sidney Pinto, 132A, Vila São Francisco, Lavras, Minas Gerais, CEP $37200-000$.

2 Universidade do Estado de Minas Gerais, UEMG - Unidade Passos, Departamento de Ciências Biomédicas e da Saúde, professora pesquisadora (orientadora). Passos, Minas Gerais, Brasil. sonia.zampieron@uemg.com. Avenida dos Expedicionários, 333, Bairro Centro, Passos, Minas Gerais, CEP 37.900-000.
} 
Os principais inimigos naturais desses insetos são os parasitoides, compreendendo o terceiro nível trófico, com predominância de espécies de Hymenoptera e, em menor escala, de Diptera (VAN DRIESCHE; BELLOWS, 1996).

De acordo com Godfray (1994), são considerados himenópteros parasitoides as espécies cujas larvas se desenvolvem no corpo de outro artrópode, geralmente um inseto, ocasionando a morte do hospedeiro ao final de seu desenvolvimento. Segundo Lasalle e Gauld (1991), esses insetos participam em mais de $50 \%$ das cadeias alimentares dos ambientes terrestres.

Uma família desse grupo estudada com frequência é a Braconidae, segunda maior família de Hymenoptera, com 29 subfamílias e cerca de 40.000 espécies em todo o mundo, aproximando-se do número global de espécies de vertebrados (ACHTERBERG, 1988; FERNANDEZ; SHARKEY, 2006). Apresentam grau variável de especificidade do hospedeiro, sendo que a maioria de suas espécies ataca insetos fitófagos, sobretudo a Lepidoptera, Diptera ou Coleoptera. Algumas espécies atacam ovos, pupas e até mesmo adultos de seus hospedeiros. O hiperparasitismo é raro. As formas adultas são de vida livre e alimentam-se de fluidos vegetais (néctar e pólen), nutrientes absorvidos, em especial na fase imatura (JERVIS et al., 1993).

O avanço expressivo de agroquímicos é considerado o principal fator do declínio da biodiversidade e abundância desses inimigos naturais, uma vez que causam intoxicação nesses organismos, podendo eliminar toda a população de insetos no agroecossistema (SILVA, 2007).

Atividades agrícolas e pecuárias têm modificado de forma contínua o Cerrado, segundo maior bioma do Brasil, que se encontra, em sua maior parte, no planalto central do país, cobrindo cerca de um quinto do território nacional (DURIGAN et al., 2004). A área total do Cerrado, que sofreu grande expansão a partir do final do Holoceno, ultrapassa 2 bilhões de km² (FURLEY, 1999).

Coutinho (2002) enfatiza que, aproximadamente, 35\% da área desse domínio brasileiro já foi transformada em pastagens cultivadas e as poucas unidades de conservação encontram-se em situação de completo abandono.

Uma das espécies vegetais muito encontrada no Cerrado é a Stryphnodendron adstringens (Mart.) Coville (Fabaceae, Mimosoidea), conhecida popularmente como Barbatimão. É uma espécie nativa, perenifólia, pioneira, anual e seletiva xerófita, característica de cerrados e campos (LORENZI, 1992; FELFILI et al., 1999). Sua ocorrência estende-se do Pará a São Paulo. Os frutos amadurecem em julho, e suas sementes são viáveis o ano todo (LORENZI, 2000).

Diante das ações antrópicas sofridas pelo Cerrado, figura a necessidade de conhecer a entomofauna local, especialmente aquela associada a frutos e sementes. O presente estudo se propôs a analisar os insetos parasitoides e suas interações com insetos endofíticos em frutos de $S$. adstringens (Mart.) Coville em uma área de Cerrado do sudoeste mineiro, localizada nas imediações do Parque Nacional da Serra da Canastra. Esse parque é a segunda maior unidade de conservação da Região Sudeste, localizando-se na porção sudoeste do estado de Minas Gerais, entre os municípios de São Roque de Minas, Delfinópolis, Sacramento, São João Batista do Glória, Capitólio e Vargem Bonita (IBDF, 1981; IBAMA, 2005).

\section{Material e métodos}

A coleta dos frutos de S. adstringens foi realizada na borda de uma estrada (20¹8'-20²9' S; 4631'-46³4' W), composta, em sua maioria, por campo sujo, campo cerrado e cerrado sensu stricto. A estrada dá acesso à área regulamentada do Parque Nacional Serra da Canastra, entre os municípios de São Roque de Minas, Delfinópolis e Sacramento (2000'-20³0' S; 46¹5'-4700' W). As coletas ocorreram uma vez por mês, de março a outubro de 2013, durante o período de fru- 
tificação de S. adstringens, totalizando oito coletas realizadas nos dias 25 de março, 17 de abril, 31 de maio, 26 de junho, 26 de julho, 29 de agosto, 26 de setembro e 21 de outubro de 2013. Foram coletados 10 frutos por exemplar vegetal; em alguns casos, a coleta se estendeu por dois ou mais exemplares, dependendo da disponibilidade de frutos de cada planta existente na área.

Os frutos eram coletados aleatoriamente em um ou vários exemplares vegetais, em alturas distintas da copa e em diferentes estágios de desenvolvimento. Tanto frutos imaturos, mas desenvolvidos, quanto os maduros e ainda fechados e os maduros iniciando a deiscência foram coletados, selecionando-se aqueles com vestígio de ataque de insetos. Para a retirada de frutos das regiões superiores da copa foram utilizados podão e tesoura de poda de haste longa (6 metros).

Uma vez coletados, todos os frutos eram conduzidos ao laboratório de Entomologia da Universidade do Estado de Minas Gerais, Unidade Passos-MG (UEMG), individualizados e acondicionados em potes plásticos de polipropileno $(750 \mathrm{ml})$, vedados com tecido de voil para contenção dos insetos emergentes, entrada de ar e para evitar a proliferação de fungos.

Todos os dias, tais frascos eram inspecionados com muito critério, sendo os adultos emergentes coletados e transferidos para frascos tipo eppendorfs, contendo álcool a 70\% para conservação, etiquetados e identificados pelos autores, de acordo com literatura especializada, a partir das chaves de identificação segundo Hanson e Gauld (2006) ou Wharton et al. (1997).

Após um período de tempo, quando não se observava mais emersão de insetos, os frutos eram abertos, a fim de localizar os insetos que não emergiram.

\section{Resultados e discussão}

Das oito coletas realizadas, de março a outubro de 2013, obteve-se um total de 210 frutos de S. adstringens (Tabela 1).

Tabela 1. Número absoluto de frutos de Stryphnodendron adstringens, coletados de março a outubro de 2013, numa área de Cerrado do Parque Nacional Serra da Canastra, MG.

\begin{tabular}{cccccccccc}
\hline Coletas & mar. & abr. & mai. & jun. & jul. & ago. & set. & out. & TOTAL \\
Frutos & 10 & 10 & 60 & 30 & 40 & 30 & 20 & 10 & 210 \\
\hline
\end{tabular}

Fonte: Elaboração dos autores

Os insetos emergidos foram totalizados em 225 indivíduos, pertencentes a sete ordens: Coleoptera (40\%), Hymenoptera (29,78\%), Lepidoptera (20,89\%), Diptera (3,55\%), Thysanoptera $(3,11 \%)$, Psocoptera (2,22\%) e Blatodea (0,44\%) (Tabela 2$)$.

Tabela 2. Ordens e número de insetos emergidos de frutos de Stryphnodendron adstringens, coletados de março a outubro de 2013, numa área de Cerrado do Parque Nacional Serra da Canastra, MG.

\begin{tabular}{cccccccccc}
\hline ORDEM & mar. & abr. & mai. & jun. & jul. & ago. & set. & out. & TOTAL \\
\hline Coleoptera & 11 & 0 & 20 & 9 & 18 & 12 & 3 & 17 & 90 \\
Hymenoptera & 18 & 0 & 22 & 8 & 11 & 2 & 0 & 6 & 67 \\
Lepidoptera & 1 & 0 & 16 & 4 & 15 & 2 & 1 & 8 & 47 \\
Diptera & 0 & 0 & 4 & 2 & 2 & 0 & 0 & 0 & 8 \\
Thysanoptera & 7 & 0 & 0 & 0 & 0 & 0 & 0 & 0 & 7 \\
Psocoptera & 5 & 0 & 0 & 0 & 0 & 0 & 0 & 0 & 5 \\
Blattodea & 0 & 0 & 0 & 0 & 0 & 1 & 0 & 0 & 1 \\
\hline TOTAL & 42 & 0 & 62 & 23 & 46 & 17 & 4 & 31 & 225 \\
\hline
\end{tabular}

Fonte: Elaboração dos autores 
A ordem com maior número de representantes foi a Coleoptera, composta, em sua maioria, por representantes da subfamília Bruchinae. Os indivíduos dessa subfamília podem consumir uma ou várias sementes durante o seu desenvolvimento, comprometendo o poder germinativo dessas. Algumas espécies constroem casulos e empupam dentro da vagem, outras empupam dentro da semente ou no solo, como no caso da espécie Acanthoscelides gregori (Pic), registrada como predadora de sementes de S. adstringens (SILVA et al., 1968; WATERWORTH, 1986; MACÊDO et al., 1992; ZIDKO, 2002; SARI; RIBEIRO-COSTA, 2011 ). Os adultos se alimentam de pólen e néctar (RIBEIRO-COSTA; ALMEIDA, 2009).

A ordem Hymenoptera, segunda mais abundante, foi representada por 11 famílias (Tabela 3), que são, em sua maioria, parasitoides de coleópteros ou lepidópteros, e de alguns exemplares de fitófagos. A biologia desses parasitoides associados a predadores de sementes ainda é pouco conhecida, sendo as principais famílias: Pteromalidae, Eulophidae (Chalcidoidea) e Braconidae (Ichneumonoidea) (DELUCA, 1980; HETZ; JOHNSON, 1988; RASPLUS, 1994), registradas também no presente trabalho.

Tabela 3. Número de himenópteros emergidos de frutos de $S$. adstringens coletados de março a outubro de 2013, numa área de Cerrado do Parque Nacional Serra da Canastra, MG.

\begin{tabular}{|c|c|c|c|c|c|c|c|c|c|}
\hline Himenóptero & mar. & abr. & mai. & jun. & jul. & ago. & set. & out. & Total \\
\hline Braconidae & 4 & 0 & 18 & 3 & 4 & 0 & 0 & 4 & 33 \\
\hline $\begin{array}{l}\text { Allorhogas sp. } \\
\text { (Doryctinae) }\end{array}$ & 0 & 0 & 12 & 2 & 0 & 0 & 0 & 1 & 15 \\
\hline $\begin{array}{l}\text { Pseudophanerotoma } \\
\text { sp. (Cheloninae) }\end{array}$ & 0 & 0 & 2 & 1 & 2 & 0 & 0 & 3 & 8 \\
\hline $\begin{array}{l}\text { Chelonus sp. } \\
\text { (Cheloninae) }\end{array}$ & 0 & 0 & 3 & 0 & 2 & 0 & 0 & 0 & 5 \\
\hline $\begin{array}{l}\text { Hormius sp. } \\
\text { (Hormiinae) }\end{array}$ & 2 & 0 & 0 & 0 & 0 & 0 & 0 & 0 & 2 \\
\hline $\begin{array}{l}\text { Bracon sp. } \\
\text { (Braconinae) }\end{array}$ & 0 & 0 & 1 & 0 & 0 & 0 & 0 & 0 & 1 \\
\hline $\begin{array}{l}\text { Apanteles sp. } \\
\text { (Microgastrinae) }\end{array}$ & 1 & 0 & 0 & 0 & 0 & 0 & 0 & 0 & 1 \\
\hline $\begin{array}{l}\text { Clinocentrus sp. } \\
\text { (Rogadinae) }\end{array}$ & 1 & 0 & 0 & 0 & 0 & 0 & 0 & 0 & 1 \\
\hline Perilampidae & 0 & 0 & 0 & 0 & 1 & 0 & 0 & 0 & 1 \\
\hline $\begin{array}{l}\text { Perilampus sp. } \\
\text { (Perilampinae) }\end{array}$ & 0 & 0 & 0 & 0 & 1 & 0 & 0 & 0 & 1 \\
\hline Ichneumonidae & 0 & 0 & 1 & 2 & 1 & 1 & 0 & 1 & 6 \\
\hline Pimplinae & 0 & 0 & 1 & 2 & 1 & 1 & 0 & 1 & 6 \\
\hline Bethylidae & 0 & 0 & 0 & 1 & 1 & 0 & 0 & 0 & 2 \\
\hline Bethylinae & 0 & 0 & 0 & 1 & 1 & 0 & 0 & 0 & 2 \\
\hline Eulophidae & 5 & 0 & 2 & 1 & 4 & 1 & 0 & 1 & 14 \\
\hline Eupelmidae & 3 & 0 & 0 & 0 & 0 & 0 & 0 & 0 & 3 \\
\hline Trichogrammatidae & 3 & 0 & 0 & 0 & 0 & 0 & 0 & 0 & 3 \\
\hline Aphelinidae & 2 & 0 & 0 & 0 & 0 & 0 & 0 & 0 & 2 \\
\hline Eurytomidae & 1 & 0 & 0 & 0 & 0 & 0 & 0 & 0 & 1 \\
\hline Pteromalidae & 0 & 0 & 0 & 1 & 0 & 0 & 0 & 0 & 1 \\
\hline Torymidae & 0 & 0 & 1 & 0 & 0 & 0 & 0 & 0 & 1 \\
\hline TOTAL & 18 & 0 & 22 & 8 & 11 & 2 & 0 & 6 & 67 \\
\hline
\end{tabular}

Fonte: Elaboração dos autores 
Quanto às famílias da ordem Hymenoptera, a melhor representada foi a Braconidae (49,25\%), com destaque para o gênero Allorhogas Gahan, incluído em Doryctinae, uma das subfamílias mais diversas desse grupo, com cerca de 1.000 espécies em regiões tropicais e subtropicais, sobretudo no Novo Mundo (WHARTON, 1997). Esses indivíduos podem ser considerados endófagos nos frutos de S. adstringens, sendo associados ao consumo das sementes. Segundo Martinez e Zaldivar-Riveron (2013), todas as espécies desse gênero de que se tem informação biológica estão associadas a galhas de plantas, tal como em tecidos de sementes de representantes das famílias Fabaceae e Melastomataceae.

Dos braconídios considerados parasitoides, houve a ocorrência dos seguintes gêneros associados aos lepidópteros: Pseudophanerotoma Zetel, Chelonus Panzer, Hormius Nees, Apanteles Foerster e Clinocentrus Haliday.

Pseudophanerotoma e Chelonus pertencem à Cheloninae, que compreende mais de 700 espécies em todo o mundo, sendo endoparasitoides solitários de ovos e formas larvais de Lepidoptera, ovipositando dentro do ovo do hospedeiro e apenas matando-o ao final do estágio larval (WHARTON et al., 1997).

Hormius (Braconidae: Hormiinae) é um gênero de ectoparasitoides gregários de lepidópteros das famílias Gelechiidae, Tortricidae e Coleophoridae. Os indivíduos Apanteles (Braconidae: Microgastrinae) são parasitoides solitários em microlepidópteros ou pouco gregários em macrolepidópteros. Clinocentrus (Braconidae: Rogadinae) é um gênero formado por parasitoides de microlepidópteros que habitam locais ocultos, tal como Tortricidae (WHARTON et al., 1997).

O único indivíduo registrado do gênero Bracon Fabricius (Braconidae: Braconinae) foi encontrado em um fruto contendo coleópteros e dípteros. De acordo com Wharton et al. (1997), os indivíduos desse gênero podem ser parasitoides de larvas de lepidópteros, coleópteros e dípteros, em particular, os que vivem escondidos em tecidos vegetais (HANSON; GAULD, 2006).

A segunda família melhor representada entre os himenópteros foi a Eulophidae (20,90\%), considerada uma das maiores dentro de Chalcidoidea, com cerca de 297 gêneros e 4.472 espécies descritas, tanto para áreas tropicais quanto para temperadas, com ampla variedade de hábitos. Algumas espécies são endo ou ectoparasitoides, idiobiontes ou cenobiontes. Podem ser solitários ou gregários, primários ou hiperparasitoides, especialistas ou generalistas e muitas espécies têm sido estudadas e usadas com sucesso em programas de controle biológico (GAUTHIER et al., 2000), sendo a terceira mais importante família de Chalcidoidea usada nesse tipo de controle (LA SALLE; SCHAUFF, 1995; SCHAUFF et al., 1997).

Os Eulophidae possuem uma ampla gama de hospedeiros, porém são parasitoides de estágios imaturos de Lepidoptera, Coleoptera, Diptera e Hymenoptera, com destaque para aqueles que vivem associados aos tecidos vegetais, como minadores, galhadores e brocas de caules (LA SALLE; SCHAUFF, 1995; SCHAUFF et al., 1997).

Em um estudo realizado por Hetz e Johnson (1988), que consta de associações de parasitoides, bruquíneos e plantas hospedeiras na América do Norte e Central, as espécies de Eulophidae Horismenus bruchophagus Burks e $H$. missouriensis (Ashmead) foram registradas associando-se ao coleóptero bruquíneo Acanthoscelides em diferentes leguminosas.

No presente estudo não foi possível determinar os hospedeiros de Eulophidae, uma vez que as amostras apresentavam representantes de coleópteros (Bruchinae), lepidópteros e de himenópteros coexistindo no mesmo fruto.

A terceira família mais abundante no estudo foi a Ichneumonidae, com seis indivíduos pertencentes à subfamília Pimplinae, que é cosmopolita e exibe uma ampla variedade em sua biologia e 
associação com hospedeiros. Podem ser ectoparasitas ou endoparasitas, idiobiontes ou cenobiontes, utilizando hospedeiros crípticos ou não. Em Pimplinae há espécies idiobiontes endoparasitas de pré-pupas ou pupas de diversos Lepidopteras (GAULD; SHAW, 1995).

De acordo com Hanson e Gaud (2006), os Pimplinae são parasitoides de Lepidoptera ou Coleoptera que vivem escondidos em plantas. Sari e Ribeiro-Costa (2011) ressaltam que, devido a dimensões maiores, é provável que espécies de Pimplinae utilizem lepidópteros como hospedeiros.

As demais famílias de Hymenoptera registradas nas coletas, tais como Elpelmidae (3 indivíduos); Trichogrammatidae (3 indivíduos); Bethylidae (2 indivíduos); Aphelinidae (2 indivíduos); Eurytomidae (1 indivíduo); Perilampidae (1 indivíduo); Pteromalidae (1 indivíduo) e Torymidae (1 indivíduo), dado o baixo número de indivíduos registrados, não foram analisadas de maneira separada quanto ao possível papel desempenhado por seus representes nesta pesquisa. A baixa expressividade desses pode estar ligada ao fato de serem parasitoides coadjuvantes nesse sistema.

Nascimento (2010) realizou um estudo em uma área de Cerrado sensu stricto da Universidade Federal de São Carlos, em São Carlos, estado de São Paulo, avaliando as interações entre insetos e frutos de espécies do local. Neste estudo foram obtidos insetos de quatro ordens: Coleoptera, Diptera, Lepidoptera e Hymenoptera. Dessa última, foram registradas oito famílias: Braconidae, Ichneumonidae, Chalcididae, Eulophidae, Eurytomidae, Perilampidae, Pteromalidae e Eupelmidae, associadas a frutos de 29 famílias de plantas. Ainda no estudo citado, os gêneros Bracon, Apanteles e Allorhogas também foram registrados em S. adstringens, assim como espécies de Calliephialtes (Ichneumonidae, Pimplinae).

Sari e Ribeiro-Costa (2011), estudando a entomofauna associada a frutos de S. adstringens coletados em diferentes fisionomias de Cerrado, no Parque Estadual do Cerrado, Jaguariaíva, Paraná, encontraram os gêneros de Braconidae: Allorhogas, Apanteles, Pseudophanerotoma e Bracon; as subfamílias Pimplinae e Bethylinae; as famílias: Eulophidae, Eurytomidae, Torymidae, Perilampidae, Chalcididae, Eupelmidae e Pteromalidae.

Com exceção da família Chalcididae, presente nos trabalhos acima citados, todas as ordens, famílias e gêneros de himenópteros ocorridos foram registrados no presente estudo.

Espécies endófagas de Allorhogas foram encontradas por Nascimento e Penteado-Dias (2005), bem como espécies de Bracon, em uma espécie de leguminosa, Stryphnodendron polyphyllum, numa área de Cerrado, em São Carlos, estado de São Paulo, e ainda espécies de Apanteles, Pseudophanerotoma e das famílias Eulophidae, Pteromalidae, Eupelmidae e Ichneumonidae associadas à $S$. adstringens, que foram registradas em frutos de $S$. adstringens nesta pesquisa.

Os indivíduos da ordem Lepidoptera encontrados no presente trabalho foram registrados em seus estágios larvais alimentando-se das sementes, sendo responsáveis, em conjunto com os coleópteros, pela maior parte da predação sofrida por essas, e por se alimentarem da polpa do fruto em que empupavam. Sari e Ribeiro-Costa (2011) registraram, em abundância, a espécie Cydia tonosticha (Meyrick) (Tortricidae) alimentando-se das sementes e da polpa dos frutos de S. adstringens em diferentes fisionomias do Cerrado no Paraná.

Quanto aos dípteros encontrados nos frutos, esses pertenciam às famílias: Cecidomyiidae (dois exemplares), principal grupo taxonômico entre os galhadores na região neotropical, respondendo por mais de 90\% das morfoespécies de galhadores (MANI, 1964; CARNEIRO et al., 2009); Sciaridae (dois exemplares), cujas larvas são conhecidas por serem fungívoras ou se alimentarem de material vegetal em decomposição (LEWANDOWSKI; SZNYK; BEDNAREK, 2004); Tachinidae (quatro exemplares), conhecidos por serem parasitoides de outros artrópodes, variando de lagartas (seus hospedeiros mais comuns) a aranhas e escorpiões (VINCENT, 1985; WILLIAMS et al., 1990). 
Neste trabalho foi possível observar resultados similares a estudos envolvendo a entomofauna presente em frutos de leguminosas no Cerrado, em especial em S. adstringens, demonstrando padrões ligados à diversidade da himenopterofauna, para a qual a família Braconidae se mostrou mais abundante.

\title{
Conclusões
}

De uma maneira geral, a entomofauna encontrada em frutos de Stryphnodendron adstringens se apresentou diversificada, estando distribuída em sete ordens de insetos.

Entre os insetos endofíticos, destacaram-se os coleópteros, por se alimentarem das sementes, e os lepidópteros, que se alimentam das sementes e da polpa do fruto. A entomofauna parasítica foi representada, em sua grande maioria, por himenópteros, havendo poucos indivíduos dípteros, sendo esses da família Tachinidae.

A família Braconidae destacou-se entre os himenópteros, sendo representada por cinco gêneros associados aos lepidópteros.

O gênero Allorhogas, considerado endófago, foi observado neste estudo.

O gênero Bracon bem como as famílias Eulophidae e Ichneumonidae não puderam ser associados a um hospedeiro específico, tendo em vista a ampla variedade em sua biologia. As famílias Eupelmidae, Trichogrammatidae, Bethylidae, Aphelinidae, Eurytomidae, Perilampidae, Pteromalidae e Torymidae apresentaram um número muito baixo de indivíduos.

Para se compreender melhor essas relações, que são pouco exploradas em pesquisas, serão necessários contínuos estudos associando os aspectos ecológicos à taxonomia dos grupos, uma vez que se torna difícil estabelecer relações tróficas concretas em nível de família.

Compreender em amplitude essas relações é de extrema importância, em especial em um bioma ameaçado, como o Cerrado. Além do mais, estudos nessa área são fundamentais para o desenvolvimento e aprimoramento de técnicas de conservação, como o controle biológico.

\section{Agradecimentos}

À Fundação de Amparo à Pesquisa do Estado de Minas - FAPEMIG, pela bolsa de iniciação científica.

Ao Instituto Nacional de Ciência e Tecnologia dos Hymenoptera Parasitoides da Região Sudeste Brasileira - INCT-HYMPAR, pela parceria e apoio logístico.

\section{Interactions between parasitoids and endophagous insects in fruits of Stryphnodendron adstringens (Mart.) Coville (Fabaceae) in Minas Gerais Cerrado.}

\begin{abstract}
Parasitoids are important controllers of phytophagous insect populations that are responsible for miscellaneous damages to the plant and could affect its survival and the seed dispersal. The biodiversity of this parasitoid fauna has been compromised by the advance of agricultural activities, especially in the Brazilian Cerrado. The objective of this study was to identify the parasitic entomofauna and their interactions with insects that feed themselves on fruits and seeds of Stryphnodendron ads-
\end{abstract}


tringens, a plant typically from the Cerrado and popularly known as Barbatimão. Monthly collections of fruits during the fruiting period were carried out, from March to October 2013 in a Cerrado area, located in the vicinity of Serra da Canastra National Park in southwestern Minas Gerais. Specimens of the Coleoptera and Lepidoptera orders were recorded as main endophagous. Among the parasitoids, the Braconidae family was noteworthy, with representatives of the genres Psedophanerotoma, Chelonus, Hormius, Apanteles and Clinocentrus associated with lepidopteran, and even samples of the genre Allorhogas, whose habit is phytophagous (endophagous). The study also showed that some Hymenoptera parasitoid families reach wide range of ecological relationships, which makes extensive studies necessary.

Keywords: Hymenoptera. Parasitoidism. Tritrophic relations. Barbatimão. Cerrado.

\section{Referências}

BARROSO, G. M.; MORIM, M. P.; PEIXOTO, A. L.; ICHASO, C. L. F.; Frutos e sementes: morfologia aplicada à sistemática de dicotiledôneas. Viçosa: UFV, 1999. 443 p.

CARNEIRO, M. A. A.; BRANCO, C. S. A.; BRAGA, C. E. D.; ALMADA, E. D.;COSTA, M. B. M.; FERNANDES, G. W.; MAIA, V. C. Are gall midge species (Diptera: Cecidomyiidae) host plant specialists? Revista Brasileira de Entomologia, v. 53, n. 3, p. 365-378, 2009.

DELUCA, Y. Catalogue des metazoaires parasites et predateurs des bruchides (Col.) (4 Note) (1). Bulletin de la Société d'Etudedes Sciences Naturelles de Nîmes, v. 86, p. 37-55, 1980.

DIGIULIO, J. A. Eurytomidae. In: GIBSON, G. A. P.; HUBER, J. T.; WOOLLEY, J. B. (Coords.). Annotated keys to the genera of Neartic Chalcidoidea (Hymenoptera). Ottawa: Ed. National Research Council, 1997. p. 477-498.

FORISTER, G. W.; JOHNSON, C. D. Behavior and ecology of Acanthoscelides prosopoides (Coleoptera: Bruchidae). The Pan Pacific Entomologist, v. 47, n. 3, p. 224-234, 1971.

FELFILI, J. M.; SILVA JÚNIOR, M. C.; DIAS, B. J.; REZENDE, A. V. Estudo fenológico de Stryphnodendron adstringens (Mart.) Coville no cerrado sensu stricto da Fazenda Água Limpa no Distrito Federal, Brasil. Revista Brasileira de Botânica, v. 22, n. 1, p. 1-14, 1999.

GAULD, I. D.; SHAW, M. E. Ichneumonidae. In: HANSON, P.; GAULD, I. (Coords.). The Hymenoptera of Costa Rica. Ed. Oxford University Press, 1995. p. 390-463.

GAUTHIER, N.; LASALLE, J.; QUICKE, D. L. J.; GODFRAY, H. C. J. Phylogeny of Eulophidae (Hymenoptera, Chalcidoidea) with a reclassification of Eulophin ae and the recognition that Elasmidae are derived eulophids. Systematic Entomology, v. 25, n. 4, p. 521-539, 2000.

GIBSON, G. A. P. Parasitic wasps of the subfamily Eupelminae: classification and revision of world genera (Hymenoptera: Chalcidoidea: Eupelmidae). Memoirs on Entomology, International, v. 5, p. 197-208, 1995a.

GIBSON, G. A. P. Eupelmidae, In: HANSON, P.; GAULD, I. (Coords.). The Hymenoptera of Costa Rica. Ed. Oxford University Press, 1995b. p. 329-336. 
GIBSON, G. A. P. Eupelmidae. In: GIBSON, G. A. P.; HUBER, J. T.; WOOLLEY, J. B. (Coords.). Annotated keys to the genera of Neartic Chalcidoidea (Hymenoptera). Ottawa: National Research Council, p. 430-476, 1997.

GODFRAY, H. C. J. Parasitoids: Behavioral an Envolutionary Ecology. Princeton: Princeton University Press, 1994. 173 p.

GRISSELL, E. E.; SCHAUFF, M. E. Chalcidoidea. In: GIBSON, G. A. P.; HUBER, J. T.; WOOLLEY, J. B. (Coords.). Annotated keys to the genera of Neartic Chalcidoidea (Hymenoptera). Ottawa: Ed. National Research Council, p.45-116, 1997.

HANSON, P. E. \& GAULD, I. (Coords). The Hymenoptera of Costa Rica. Ed: Oxford University Press, 1995.

HANSON, P. E. Torymidae. In: HANSON, P. E.; GAULD, I. (Coords.). The Hymenoptera of Costa Rica. Ed. Oxford University Press, p. 377-383. 1995b.

HANSON, P. E.; GAULD, I. D. (Eds). Hymenoptera de La Region Neotropical. Memoirs of the American entomological Institute, v. 77, n. 1, 994 p. 2006.

HETZ, M.; JOHNSON, C. D. Hymenopterous parasites of some bruchid beetles in North and Central America. Journal of Stored Products Research, v. 24, p. 131-134, 1988.

HINCKLEY, A. D. Comparative ecology of two beetles established in Hawaii: an Anthribid, Araecerus levipennis, and a bruchid, Mimoses tessallaei. Ecology, v. 42, n. 3, p. 526-532, 1961.

IBAMA. Plano de Manejo. Parque Nacional Serra da Canastra. IBAMA, 2005. 828 p.

JANZEN, D. H. Ecologia vegetal nos trópicos. São Paulo: EPU; EDUSP, 1980. 76 p.

LASALLE, J.; GAULD, I. D. Parasitic hymenoptera and the biodiversity crisis. Redia, v. 74, n. 3, p. 315-334, 1991.

LASALLE, J.; SCHAUFF, M. E. Eulophidae, The Braconidae. In: HANSON, P.; GAULD, I. (Coords.). The Hymenoptera of Costa Rica. Ed. Oxford University Press, p. 315-329, 1995.

LEWANDOWSKI, M.; SZNYK, A.; BEDNAREK, A. Biology and morphometry of Lycoriellaingenua (Diptera: Sciaridae). Biology Letters, v. 41, n. 1, p. 41-50, 2004.

LEWIS, T. Thrips, their biology, ecology and economic importance. London, Academic Press, 1973. $349 \mathrm{p}$.

LORENZI, H. Árvores brasileiras: manual de identificação e cultivo de plantas arbóreas nativas do Brasil. São Paulo: Ed. Plantarum, 1992. 352 p.

LORENZI, H. Árvores brasileiras: manual de identificação e cultivo de plantas arbóreas nativas do Brasil. Nova Odessa: Editora, 2000. 325 p.

MACÊDO, M. V.; LEWINSOHN, T. M.; KINGSOLVER J. M. New host records of some bruchid species in Brazil with the description of a new species of Caryedes (Coleoptera: Bruchidae). Coleopterists Bulletin, v. 46, n. 4, p.330-336, 1992. 
MANI, M. S., Ecology of plant galls. W. Junk Publisher, Netherlands, 1964. 434 p.

MARTINEZ, J. J.; ZALDIVAR-RIVERON, A. Seven new species of Allorhogas (Hymenoptera: Braconidae: Doryctinae) from Mexico. Revista Mexicana de Biodiversidad, México, v. 84, n. 1, 2013.

MOUND, L. A.; MARULLO, R. The thrips of Central and South America: an introduction (Insecta: Thysanoptera). Mem. Entomol. Inter. v. 6, 1996. 488 p.

NASCIMENTO, A. G. Estudo de interações de parasitoides de insetos endófagos em frutos do Cerrado. 2010. 59 f. Dissertação (Mestrado em Ecologia e Recursos Naturais), Universidade Federal de São Carlos, São Carlos, 2010.

NASCIMENTO, A. G.; PENTEADO-DIAS, A. M. A fauna de hymenoptera associada às espécies de leguminosas que ocorrem em área de cerrado. In: Reunião Anual do Instituto Biológico, 18. ed., 2005, São Paulo. Arquivos do Instituto Biológico, v. 72, (supl.2), São Paulo: Instituto Biológico, p. 63. 2005.

OTT, J. R. The biology of Acanthoscelides alboscutellatus (Coleoptera: Bruchidae) on its host plant, Ludwigia alternifolia (L.) (Onagraceae). Proceedings of the Entomological Society of Washington, $v$. 93, n. 2, p. 641-651, 1991.

PINTO, J. D. Trichogrammatidae, In: HANSON, P.; GAULD, I. (Coords). The Hymenoptera of Costa Rica. Oxford: Oxford University Press, 1995. p. 383-388.

PINTO, J. D. Trichogrammatidae, In: GIBSON, G. A. P.; HUBER, J. T.; WOOLLEY, J. B. (Coords.). Annotated keys to the genera of Neartic Chalcidoidea (Hymenoptera). Ottawa: Ed. National Research Council, p. 726-752, 1997.

RASPLUS, J. Y. Parasitoid communities associated with west African seed-feeding beetles, In: BRADFORD, A.; HAWKINS, S.; SHEEHAN, W. Parasitoid community ecology. Oxford: Oxford University press, p. 319-342, 1994.

RIBEIRO-COSTA, C. S.; ALMEIDA, L. M. Bruchinae (Coleoptera: Chrysomelidae). In: PANIZZI, A.; PARRA, J. R. P. (Coords). Bioecologia e Nutrição de Insetos: Base para o manejo Integrado de Pragas. Brasília: Ed. Embrapa Informação Tecnológica, p. 523-567, 2009.1164p.

SARI, L. T.; RIBEIRO-COSTA, C. S. Entomofauna associada aos frutos de Stryphnodendron adstringens (Mart.) Coville (Mimosoideae) no Parque Estadual do Cerrado, Jaguariaíva, Paraná. Coletânea de Pesquisa do Parque Estadual de Vila Velha, Cerrado e Guartelá. Curitiba. v. 1, n. 1, p.316-325, 2011.

SCHAUFF, M. E.; COOTE, L. D. Eulophidae, In: GARY, A.; GIBSON, P.; HUBER, J. T.; WOOLLEY, J. B. (Coords). Annotated keys to the genera of Neartic Chalcidoidea (Hymenoptera). Ottawa: Ed. National Research Council, p. 327-429, 1997.

SILVA, A. G. D.; GONÇALVEZ, C. R.; GALVÃO, D. M.; GONÇALVES, A. J. L.; GOMES, J.; SILVA, M. N.; SIMONI, L. Quarto catálogo dos insetos que vivem nas plantas do Brasil, seus parasitos e predadores. Rio de Janeiro: Ed. Ministério da Agricultura, 1968.

SILVA, A. F. Seleção, biologia e exigências térmicas de Trichogramma sp. criados em ovos de Diaphania nitidalis Cramer (Lepidoptera: Pyralidae). 2007. 44 f. Dissertação (Mestrado em Produção Vegetal), Centro de Ciências Agrárias, Universidade Federal do Espírito Santo, Alegre, 2007. 
VINCENT, L. S. The firstrecordof a tachinidfly as aninternalparasitoidof a spider (Diptera: Tachinidae; Araneae: Antrodiaetidae). Pan-Pacific Entomologist, v. 61, p. 224-225, 1985.

WATERWORTH, P. D. Internal seed infesting insects. United States Department of Agriculture -Animal and Plant Health Inspection Service. 1986.

WHARTON, R. A. Introduction, In: WHARTON, R. A.; MARSH, P. M.; SHARKEY, M. J. (Coords.). Manual of the New World Genera of the Family Braconidae (Hymenoptera). Ed. International Society of Hymenopterists. Special publication 1, p. 1-16, 1997.

WHARTON, R. A.; MARSH, P. M.; SHARKEY, M. J. (Coords). Manual of the New World Genera of the Family Braconidae (Hymenoptera). Ed. International Society of Hymenopterists. Special publication 1, 1997. 439 p.

WILLIAMS, S. C., ARNAUD, P. H.Jr.; LOWE, G. Parasitism of Anuroctonus phaiodactylus (Wood) and Vaejovis spinigerus (Wood) (Scorpiones: Vaejovidae) by Spilochaetosoma californicum Smith (Diptera: Tachinidae) and a review of parasitism in scorpions. Myia, v. 5, p. 11-27, 1990.

ZHANG, J.; DRUMMOND, F. A.; LIEBMAN, N; HATKE, A. Insect predation of seeds and plant population dynamics. Technical Bulletin 163, University of Maine, Maine Agricultural and Forest Experimentation, $1997,32 \mathrm{p}$.

ZIDKO, A. Coleópteros (Insecta) associados às estruturas reprodutivas de espécies florestais arbóreas nativas no Estado de São Paulo. 2002. 135 f. Dissertação (Mestrado), Escola Superior de Agricultura "Luiz de Queiroz"/ Universidade de São Paulo, São Paulo, 2002.

\section{Histórico editorial}

Submetido em: 05/09/2014

Aceito em: 02/06/2015 